\title{
Minimal clinically important differences in nasal peak inspiratory flow*
}

\author{
Daniel Timperley ${ }^{1,3}$, Aviva Srubisky², Nicholas Stow ${ }^{4}$, George N. Marcells ${ }^{3}$, \\ Richard J. Harvey ${ }^{1}$ \\ 1 Department of Otolaryngology / Skull Base Surgery, St. Vincent's Hospital, Darlinghurst, \\ Sydney NSW 2010, Australia \\ 2 School of Medicine, University of Sydney NSW 2006, Australia \\ 3 Bondi Junction Private Hospital, Bondi Junction NSW 2022, Australia \\ 4 Mona Vale Hospital, Mona Vale NSW 2103, Australia
}

SUMMARY

\begin{abstract}
Introduction: Acoustic Rhinometry, Rhinomanometry, Nasal Spirometry and Nasal Peak Inspiratory flow (NPIF) all measure subtly different constructs of nasal function. All have limitations but NPIF is simple and quick to integrate into clinical practice. The minimum clinically important difference (MCID) for an outcome measure is an estimate of the smallest change that is experienced by a patient or group as being significant. Studies, particularly with large samples, may generate results that while statistically significant, have limited clinical effect. Defining MCID allows an assessment of the clinical impact of an intervention. This study defines the MCID for NPIF.

Methods: Prospective study of patients from a tertiary clinic undergoing open septorhinoplasty. Nasal obstruction scores and NPIF were recorded before and after surgery. Global function and nasal obstruction scores were used to assess subjective change. Statistical based and patient anchored techniques were used to define MCID.

Results: 51 patients with a mean age $36 \pm 13$ yrs (75\% female) were recruited. All had open rhinoplasty, septal reconstruction, spreader grafts and turbinate reduction. Baseline NPIF was $101 \pm 35 \mathrm{~L} / \mathrm{min}$. The statistically derived MCID (half standard deviation) was $18 \mathrm{~L} / \mathrm{min}$, the patient anchored approaches were $20 \mathrm{~L} / \mathrm{min}$ and 20-25 L/min.

Discussion: Although NPIF is effort dependant with the potential for poor test-retest reliability, it is simple, quick and a reliable technique can be quickly learnt. An MCID of 20L/min is recommended when NPIF is used as an outcome tool. Understanding the MCID is critical for assessing the impact of nasal surgery.
\end{abstract}

Key words: Outcome assessment, nasal obstruction, treatment outcome, rhinoplasty, turbinates, nasal septum, respiratory function tests, rhinomanometry

\section{INTRODUCTION}

Measurement of the nasal airway allows objective assessment of nasal obstruction and quantification of the effects of medical or surgical interventions. Measurement methods can be grouped into anatomical (eg. acoustic rhinometry), physiological (eg. rhinomanometry, spirometry, nasal peak inspiratory flow (NPIF)) and subjective (questionnaire based tools) measures. Outcomes do not necessarily correlate well between the three categories, with all groups measuring subtly different aspects of nasal function ${ }^{(1,2)}$. Nasal peak inspiratory flow is a physiological measure of nasal airflow which is particularly sensitive to nasal valve collapse. It is reproducible and has been validated against rhinomanometry ${ }^{(1,3,4-6)}$. It requires only simple equipment and is easy to perform, making it practical for routine use in the office/clinic environment.

The minimal clinically important difference (MCID) of an outcome measure is the smallest difference that is considered to be worthwhile or important ${ }^{(7)}$. Difference in outcome measures, particularly in large samples, may reach statistical significance despite the magnitude of the effect being small and possibly not clinically significant. Knowledge of the MCID for an outcome measure allows an assessment of the clinical impact of an intervention. Methods for determining MCID can be classified into distributional (statistical), anchor (external measure) and opinion based approaches. The different approaches tend to

Presented at: 63rd NZSOHNS Annual General and Scientific Meeting; 2-5 March 2010; Bay of Islands, New Zealand. ASOHNS 2010 Diamond Jubilee Annual Scientific Meeting; 27-30 March 2010; Sydney, Australia 
converge on the same answers, and it has been suggested that the most useful MCID may be one that is the product of multiple approaches ${ }^{(8-12)}$. This study aims to determine the MCID of NPIF from a cohort of patients undergoing external septorhinoplasty.

\section{METHODS}

\section{Study population}

Data was gathered prospectively in a cohort of patients undergoing external septorhinoplasty. The surgical technique included spreader grafts and endoscopic turbinoplasty. Recruitment was between February 2007 and December 2008. Ethics approval was obtained through the local hospital institutional review board.

\section{Outcome measures}

The outcomes assessed were NPIF, nasal obstruction scores and patient anchor scores. Patients were reviewed preoperatively, 1 week, 3 weeks, 12 weeks and either 3 or 6 monthly thereafter. The baseline and last follow up were used for assessment.

The NPIF is measured in a seated position using a mini-Wright peak flow meter with an anaesthetic mask (Figure 1). A good seal is ensured and the patient instructed to make a maximal inspiratory effort with the mouth closed. The best result of three attempts is used after appropriate training ${ }^{(5,3)}$.

Patient anchor scores were assessed at follow-up on a 7 point Likert scale. Patients were asked to rate their change in nasal function from -3 (significant deterioration) to +3 (significant improvement), with 0 representing no change.

Subjective nasal obstruction was assessed using a Likert scale of $0-5$, with 0 representing no obstruction and 5 as bad as it can be. This was undertaken at baseline and during all followup visits.

\section{Analysis}

According to the taxonomy of responsiveness described by Beaton et al. ${ }^{(9)}$, this study aims to calculate the MCID for NPIF using patient based measures of within-person change over time. Both distributional and anchor based approaches

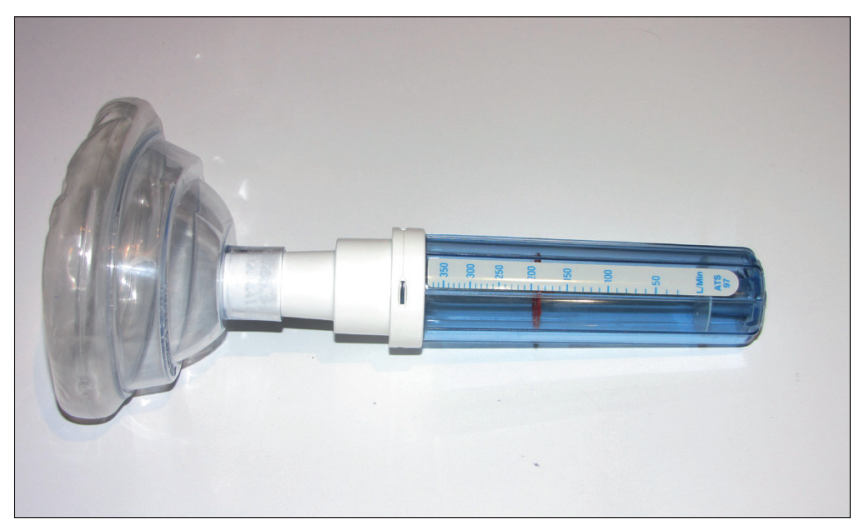

Figure 1. Mini Wright peak flow meter used for measurement of NPIF. NPIF is measured in a sitting position, with the mask applied to ensure a good seal without compressing the external nares. were used. Statistical analysis was with SPSS v17 (Statistical software for social sciences, SPSS Inc. Chicago, IL, USA).

Anchor based

Anchor based approaches use an external measure, known as the anchor. The use of global function scores as an external anchor is a well established method for establishing MCID. The classic approach, described by Jaeschke et al., used a 15 point Likert scale, however, others subsequently have used a 7 point scale, as undertaken in this study ${ }^{(13-15)}$. Global function scores were used as the primary anchor in this study.

Change in nasal obstruction score over time (pre vs postoperative) was used as a second external anchor. This may provide an overestimate of the MCID for NPIF, because while the minimum change possible in obstruction score is one point, the MCID for similar Likert scales is likely to be less than one (14,16). We therefore also assessed the MCID for the obstruction scores using the distributional method described below.

For each external anchor, correlation between the anchor and NPIF was confirmed with Spearman's test. The mean change in NPIF was compared for different values of the anchor score.

\section{Distributional}

The MCID can be assessed by estimation of the standard error of the mean (SEM). SEM is estimated by the standard deviation of the measurement multiplied by the square root of one minus it's reliability coefficient, or A related approach, which has shown to be valid for quality of life measures, is the use of

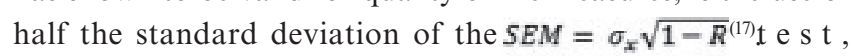
which corresponds to a reliability coefficient of $0.75^{(8,16)}$. Both of these approaches have been primarily applied to questionnaire based outcome measures rather than objective measures. In this study, the reliability coefficient for NPIF is not known, therefore the $0.5 \mathrm{SD}$ method has been used. The MCID calculated by these methods was compared to that calculated from the anchor based methods.

\section{RESULTS}

\section{Baseline characteristics}

Fifty-one patients with a mean age of 36 and $75 \%$ female were recruited. The mean follow-up duration was 8.5 months (range 4.8-19.1). Baseline NPIF was $101 \pm 35 \mathrm{~L} / \mathrm{min}$ and median obstruction score was 3 (interquartile range 2-4). At followup, mean NPIF improved to $143 \pm 44 \mathrm{~L} / \mathrm{min}$, with 44 patients improved and 7 unchanged or worse. The median postoperative obstruction score was 0 (interquartile range 0-1) with 39 patients improved, 11 unchanged and one worse.

\section{Anchor-based: Global function score}

A score of plus or minus one or more was considered to be clinically significant, however, as there was only one patient with a score of +1 , scores of +1 and +2 were grouped for statistical purposes. There was only one patient with a negative score, therefore meaningful analysis for negative scores was not possible. There was a significant correlation between the 


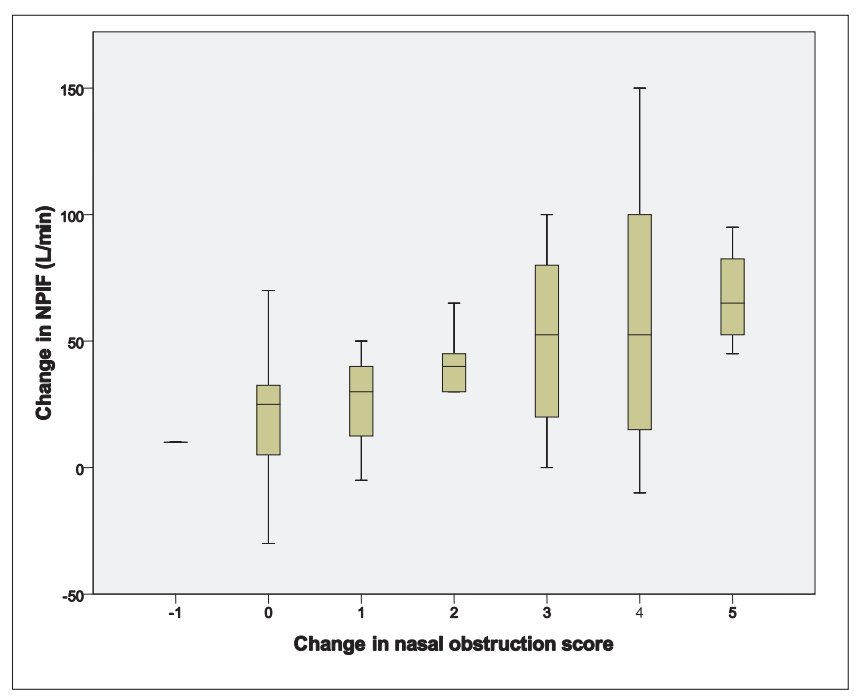

Figure 2. Comparison of change in NPIF with change in obstruction score.

global function score and the change in NPIF (Spearman's R $=0.56, p<0.01$ ). Patients reporting no change (global function score of zero) had a mean increase in NPIF of $14 \pm 10 \mathrm{~L} /$ min whereas patients who reported slight / moderate improvement $(+1 /+2)$ had a mean increase in NPIF of $20 \pm 7 \mathrm{~L} / \mathrm{min}$ and those who reported a significant improvement had mean increase in NPIF of $58 \pm 6 \mathrm{~L} / \mathrm{min}$. This data suggests a value for the MCID of $20 \mathrm{~L} / \mathrm{min}$.

\section{Anchor based: change in obstruction score}

There was significant correlation between the change in obstruction score and the change in NPIF (Spearman's R = $0.43, \mathrm{p}<0.01)$. A comparison of the average change in NPIF with changes in obstruction score is given in Table 1 and Figure 2. Application of the half standard deviation distributional method to our obstruction score data gives a MCID for the obstruction score of 0.75 . Therefore, using a change of one point is likely to give an overestimate of the MCID for NPIF. From inspection of the data (Table 1), the MCID is likely to be between 20-25 L/minute.

\section{Distributional}

The standard deviation NPIF was $35.4 \mathrm{~L} /$ minute at baseline, therefore the MCID calculated using this method, as a half

Table 1. Comparison of change in obstruction score with change in NPIF. The MCID for change in obstruction score was 0.7 (statistically derived). Therefore, the MCID for NPIF is likely to lie between 20 and $25 \mathrm{~L} / \mathrm{min}$.

\begin{tabular}{cc}
\hline Change in obstruction score & Change in NPIF (L/min) \\
\hline 0 & $20 \pm 8$ \\
1 & $25 \pm 16$ \\
2 & $41 \pm 4$ \\
3 & $49 \pm 9$ \\
4 & $59 \pm 17$ \\
5 & $67 \pm 11$ \\
\hline
\end{tabular}

standard deviation, is $18 \mathrm{~L} / \mathrm{min}$.

\section{DISCUSSION}

The MCID for NPIF of $20 \mathrm{~L} / \mathrm{min}$, estimated from the global function scores, is supported by the other two methods. The estimate from the change in obstruction score is slightly higher, lying between $20-25 \mathrm{~L} / \mathrm{min}$, while that from the distributional method is slightly lower, at $18 \mathrm{~L} / \mathrm{min}$. We therefore propose an MCID for NPIF of $20 \mathrm{~L} / \mathrm{min}$.

Methods for measurement of nasal flow can be grouped into anatomic (e.g. acoustic rhinometry), physiologic (e.g. NPIF, rhinomanometry) and subjective (e.g. VAS, Likert scales). Although Lam et al. demonstrated no significant correlations between measures of nasal airflow from different groups (18), other authors have demonstrated correlations between different categories of measurement ${ }^{(5,19-21)}$. It is likely that different patient groups will demonstrate varying degrees of correlation as the measurement assess different aspects of nasal physiology.

The use of NPIF in assessment has been well examined. A normal value of greater than $120 \mathrm{~L} / \mathrm{min}$ is commonly used ${ }^{(22,23)}$. NPIF is affected by nasal wall compliance in addition to nasal patency, making it sensitive to nasal valve collapse ${ }^{(24,25)}$. NPIF is effort dependent and also affected by lower respiratory function but is not affected by height, weight or gender ${ }^{(3,6)}$. It's reproducibility has been questioned by some researchers ${ }^{(26-28)}$. However, others have found it to be readily reproducible and correlate well with rhinomanometry ${ }^{(1,5)}$ and subjective measures ${ }^{(5,20,21)}$. Patient training and education is essential. Changes in NPIF measurement may result from leakage of air through the mouth or fatigue. If the anaesthetic mask is too small or not positioned appropriately, it can compress the external nares, whereas if it is too large, air leak may result (25). Careful instruction is required to ensure good technique, which is vital to achieve reproducible results. Most patients are able to achieve reproducible results with less than 5 minutes instruction ${ }^{(1)}$. Taking the best of three readings (rather than the mean) with less than $10 \%$ variation is suggested ${ }^{(25)}$. NPIF is easily incorporated into clinical practice, requiring inexpensive equipment and able to be performed rapidly in comparison to the bulky, expensive equipment required for rhinomanometry and acoustic rhinometry.

The concept of MCID, and the difference between statistical and clinical significance is important in interpreting outcome data. For example, a treatment may be shown to alter an outcome in $80 \%$ of a patient group. However, if only $10 \%$ of the group have a change greater than the MCID of that outcome measure, the remainder of the patients will not experience a meaningful change, whether positive or negative. Where the MCID is known, it should be reflected in reported results.

\section{CONCLUSIONS}

Nasal peak inspiratory flow is a simple, inexpensive physiologic test of nasal patency that is easily incorporated into clinical practice. Estimation of the minimal clinically important difference for this outcome tool allows more clinically relevant 
assessment of outcomes data. An MCID for NPIF of 20L/ minute is proposed.

\section{ACKNOWLEDGEMENTS}

No external funding was received for this study.

\section{AUTHORSHIP CONTRIBUTION}

DT: Design, implementation and manuscript preparation; AS: data collection, manuscript review; NS: data collection, manuscript review; GM: design, implementation and manuscript review; RH: design, implementation and manuscript review.

\section{CONFLICT OF INTEREST}

Dr. Harvey, has served on an advisory board for Schering Plough and has received grant support from NeilMed. Daniel Timperley, Aviva Srubisky, Nicholas Stow and George Marcells have no conflicts of interest to declare.

\section{REFERENCES}

1. Holmström M, Scadding GK, Lund VJ, et al. Assessment of nasal obstruction. A comparison between rhinomanometry and nasal inspiratory peak flow. Rhinology 1990; 28: 191-196.

2. Hopkins C. Patient reported outcome measures in rhinology. Rhinology 2009; 47: 10-17.

3. Starling-Schwanz R, Peake HL, Salome CM, et al. Repeatability of peak nasal inspiratory flow measurements and utility for assessing the severity of rhinitis. Allergy 2005; 60: 795-800.

4. Wihl JA, Malm L. Rhinomanometry and nasal peak expiratory and inspiratory flow rate. Ann Allergy 1998; 61: 50-55.

5. Jones AS, Viani L, Phillips D, et al. The objective measurement of nasal patency. Clin Otolaryngol 1991; 16: 206-211.

6. Phagoo SB, Watsoti RA, Pride NB. Use of nasal peak flow to assess nasal patency. Allergy 1997; 52: 901-908.

7. Hays RD, Woolley JM. The concept of clinically meaningful difference in health-related quality-of-life research. How meaningful is it? Pharmacoeconomics 2000; 18: 419-423.

8. Sloan JA. Assessing the minimally clinically significant difference: scientific considerations, challenges and solutions. COPD. [Review]. 2005; 2: 57-62.

9. Beaton DE, Boers M, Wells GA. Many faces of the minimal clinically important difference (MCID): a literature review and directions for future research. Curr Opin Rheumatol. [Review]. 2002; 14: 109-114.

10. Samsa G, Edelman D, Rothman ML, et al. Determining clinically important differences in health status measures: a general approach with illustration to the Health Utilities Index Mark II. Pharmacoeconomics 1999; 15: 141-155.

11. Kvien TK, Heiberg T, Hagen KB. Minimal clinically important improvement/difference (MCII/MCID) and patient acceptable symptom state (PASS): what do these concepts mean? Ann Rheum Dis. [Review]. 2007; 66 Suppl 3: 40-41.

12. Make B. How can we assess outcomes of clinical trials: the MCID (ap) roach. COPD. [Review]. 2007; 4: 191-194.

13. Lang CE, Edwards DF, Birkenmeier RL, et al. Estimating minimal clinically important differences of upper-extremity measures early after stroke. Arch Phys Med Rehabil. 2008; 89: 1693-1700.

14. Jaeschke R, Singer J, Guyatt GH. Measurement of health status. Ascertaining the minimal clinically important difference. Control Clin Trials 1989; 10: 407-415.

15. Copay AG, Subach BR, Glassman SD, et al. Understanding the minimum clinically important difference: a review of concepts and methods. Spine J. [Review]. 2007; 7: 541-546.

16. Norman GR, Sloan JA, Wyrwich KW. Interpretation of changes in health-related quality of life: the remarkable universality of half a standard deviation. Med Care. 2003; 41: 582-592.

17. Wyrwich KW, Tierney WM, Wolinsky FD. Further evidence supporting an SEM-based criterion for identifying meaningful intra-individual changes in health-related quality of life. J Clin Epidemiol. 1999; 52: 861-873.

18. Lam DJ, James KT, Weaver EM. Comparison of anatomic, physiological, and subjective measures of the nasal airway. Am J Rhinol 2006; 20: 463-470.

19. Mora F, Cassano M, Mora R, et al. V.A.S. in the follow-up of turbinectomy. Rhinology 2009; 47: 450-453.

20. Jose J, Ell SR. The association of subjective nasal patency with peak inspiratory nasal flow in a large healthy population. Clin Otolaryngol 2003; 28: 352-354.

21. Fairley JW, Durham LH, Ell SR. Correlation of subjective sensation of nasal patency with nasal inspiratory peak flow rate. Clin Otolaryngol 1993; 18: 19-22.

22. Ottaviano G, Scadding GK, Coles S, et al. Peak nasal inspiratory flow; normal range in adult population. Rhinology 2006; 44 : 32-35.

23. Bermuller C, Kirsche H, Rettinger G, et al. Diagnostic accuracy of peak nasal inspiratory flow and rhinomanometry in functional rhinosurgery. Laryngoscope 2008; 118: 605-610.

24. Fodil R, Brugel-Ribere L, Croce C, e al. Inspiratory flow in the nose: a model coupling flow and vasoerectile tissue distensibility. $\mathrm{J}$ Appl Physiol 2005; 98: 288-295.

25. Nathan RA, Eccles R, Howarth PH, et al. Objective monitoring of nasal patency and nasal physiology in rhinitis. J Allergy Clin Immunol 2005; 115: S442-S459.

26. Gleeson MJ, Youlten LJ, Shelton DM, et al. Assessment of nasal airway patency: a comparison of four methods. Clin Otolaryngol 1986; 11: 99-107.

27. Larsen K, Kristensen S. The peak flow nasal patency index. Ear Nose Throat J 1992; 71: 23-25.

28. Enberg RN, Ownby DR. Peak nasal inspiratory flow and Wright peak flow: a comparison of their reproducibility. Ann Allergy $1991 ; 67: 371-374$

Daniel Timperley MBChB, FRACS

Hospitals C. Yincent's and Bondi Junction Private Sydney, 354 Victoria St

Darlinghurst

NSW 2010, Sydney

Australia

Tel: +61-2-9360 4811

Mobile: +61-4-5054 2387

Fax: +61-2-9360 9919

E-mail: daniel.timperley@gmail.com 